\title{
LOCATION OF OBJECTS WITH A HIGH DEGREE OF INNER PRECISION
}

\author{
LUBOMÍR KUBÁČEK ${ }^{1}$
}

\section{Abstract}

Let us have two coordinate systems in a plane. In the first one (e.g. JTSK) the accuracy of the coordinate estimators is signicantly lower than the accuracy in the other system (the inner system of an object). The task is to determine the coordinate estimators of the characteristic points of the object in the first system (with a signicantly lower degree of accuracy) in such a way that the relative accuracy of the characteristic points is the same as the relative accuracy in the inner system.
Address

1 Department of Mathematical Analysis and Applications of Mathematics, Faculty of Science, Palacký University, Tř. 17. listopadu č. 12, CZ-771 00 Olomouc, Czech Republic

Corresponding author: lubomir.kubacek@upol.cz

Keywords

- Best linear unbiased estimator (BLUE),

- linear conform

- transformation

\section{INTRODUCTION}

The location of every new engineering work (a nuclear plant, tunnel, dam, etc.) must be determined in a state coordinate system, i.e., the coordinates of the characteristic points of the structure must be determined. The requirements for precision in a state coordinate system are usually less demanding than the requirements in the internal system of the new structure. In the following text, for the sake of simplicty, it will be assumed that the internal system is error-free. Corresponding problems see also in [2], [3], [5], [7].

Thus the task is to nd such coordinate estimators of the characteristic points in the state system so that the dispersions of the estimators of the distances among the characteristic points are zeros.

\section{NOTATION}

Let $\mathbf{a}=\left(\mathbf{a}_{1}^{\prime}, \ldots, \mathbf{a}_{k}^{\prime}\right)^{\prime}$ be the coordinate vector of the characteristic points $P_{l}, \ldots, P_{k}$, of the new structure, $\mathbf{a}_{i}$ be the $2 \mathrm{D}$ coordinate vector of the $i$-th characteristic point $P_{i}$, and $i=1, \ldots, k$. Let $\widehat{\Theta}$ be the estimator of the coordinates of points $B_{1}, \ldots, B_{N}$, in the state coordinate system in the neigh- bourhood of the new structure, which enables us to determine the state coordinates of the characteristic points $P_{1}, \ldots, P_{k}$. The fact that the mean value $E(\widehat{\Theta})$ of the estimator $\widehat{\Theta}$ is $\Theta$ and its covariance matrix $\operatorname{Var}(\widehat{\Theta})$ is $W$, is denoted as $\widehat{\Theta} \sim_{2 N}(\Theta, W)$. The real value of the coordinate vector of the point $P_{i}$ in the state coordinate system is $\mathbf{b}_{i}$. The preliminary estimator $\tilde{b}_{i}$ of the vector $\mathbf{b}_{i}$ is based on the observation vector $\mathbf{Y} \sim_{n}(\mathrm{D} \Theta+\mathrm{Fb}, \Sigma)$, on the vector $\widehat{\Theta}$ and on the covariance matrix $\mathbf{W}$. The $n \times 2 k$ matrix $\mathbf{F}$ is the design matrix of a measurement. The $n \times 2 N$ matrix $\mathbf{D}$ is the incidental matrix which characterizes the connection of the points $B_{1}, \ldots, B_{N}$ with the points $P_{1}, \ldots, P_{k}$. The covariance matrix $\Sigma$ usually does not satisfy the requirements for the accuracy of the internal points of the structure; however, it satisfies the requirement for the accuracy of the state system.

\section{ESTIMATOR OF THE VECTOR b}

Lemma 2.1 Let the rank of the matrix $\mathbf{F}$ be $r(\mathbf{F})=2 k<n$, and let $\mathbf{W}$ and $\boldsymbol{\Sigma}$ be positive definite matrices. The best linear unbiased estimator (BLUE) of the vector $\mathbf{b}$ in the model

$$
\left(\begin{array}{c}
\widehat{\Theta} \\
\mathbf{Y}
\end{array}\right) \sim_{2 N+n}\left[\left(\begin{array}{cc}
\mathbf{I}, & \mathbf{0} \\
\mathbf{D}, & \mathbf{F}
\end{array}\right)\left(\begin{array}{l}
\boldsymbol{\Theta} \\
\mathbf{b}
\end{array}\right),\left(\begin{array}{cc}
\mathbf{W}, & \mathbf{0} \\
\mathbf{0}, & \mathbf{\Sigma}
\end{array}\right)\right]
$$

is

$$
\tilde{\mathbf{b}}=\left[\mathbf{F}^{\prime}\left(\boldsymbol{\Sigma}+\mathbf{D W D}^{\prime}\right)^{-1} \mathbf{F}\right]^{-1} \mathbf{F}^{\prime}\left(\boldsymbol{\Sigma}+\mathbf{D W D}^{\prime}\right)^{-1}(\mathbf{Y}-\mathbf{D} \widehat{\boldsymbol{\Theta}}) .
$$

$\operatorname{Var}(\tilde{\mathbf{b}})=\left[\mathbf{F}^{\prime}\left(\boldsymbol{\Sigma}+\mathbf{D W D}^{\prime}\right)^{-1} \mathbf{F}\right]^{-1}$ 
Proof. The BLUE $\tilde{\mathbf{b}}$ in this model is

$$
\begin{gathered}
\tilde{\mathbf{b}}=\left(\mathbf{0}_{2 k, 2 N}^{\prime}, \mathbf{I}_{2 k}\right)\left[\left(\begin{array}{cc}
\mathbf{I}_{2 N}, & \mathbf{D}^{\prime} \\
\mathbf{0}, & \mathbf{F}^{\prime}
\end{array}\right)\left(\begin{array}{cc}
\mathbf{W}^{-1}, & \mathbf{0} \\
\mathbf{0}, & \boldsymbol{\Sigma}^{-1}
\end{array}\right)\left(\begin{array}{cc}
\mathbf{I}, & \mathbf{0} \\
\mathbf{D}, & \mathbf{F}
\end{array}\right)\right]^{-1} \\
\times\left(\begin{array}{cc}
\mathbf{I}_{2 N}, & \mathbf{D}^{\prime} \\
\mathbf{0}, & \mathbf{F}^{\prime}
\end{array}\right)\left(\begin{array}{cc}
\mathbf{W}^{-1}, & \mathbf{0} \\
\mathbf{0}, & \boldsymbol{\Sigma}^{-1}
\end{array}\right)\left(\begin{array}{c}
\widehat{\boldsymbol{\Theta}} \\
\mathbf{Y}
\end{array}\right) \\
=\left(\mathbf{0}^{\prime}, \mathbf{I}\right)\left(\begin{array}{cc}
\mathbf{W}^{-1}+\mathbf{D}^{\prime} \boldsymbol{\Sigma}^{-1} \mathbf{D}, & \mathbf{D}^{\prime} \boldsymbol{\Sigma}^{-1} \mathbf{F} \\
\mathbf{F}^{\prime} \boldsymbol{\Sigma}^{-1} \mathbf{D}, & \mathbf{F}^{\prime} \boldsymbol{\Sigma}^{-1} \mathbf{F}
\end{array}\right)\left(\begin{array}{c}
\mathbf{W}^{-1} \widehat{\boldsymbol{\Theta}}+\mathbf{D}^{\prime} \boldsymbol{\Sigma}^{-1} \mathbf{Y} \\
\mathbf{F}^{\prime} \boldsymbol{\Sigma}^{-1} \mathbf{Y}
\end{array}\right) \\
=(21,22)\left(\begin{array}{c}
\mathbf{W}^{-1} \widehat{\boldsymbol{\Theta}}+\mathbf{D}^{\prime} \boldsymbol{\Sigma}^{-1} \mathbf{Y} \\
\mathbf{F}^{\prime} \boldsymbol{\Sigma}^{-1} \mathbf{Y}
\end{array}\right),
\end{gathered}
$$

where

$$
\left(\begin{array}{cc}
\mathbf{W}^{-1}+\mathbf{D}^{\prime} \boldsymbol{\Sigma}^{-1} \mathbf{D}, & \mathbf{D}^{\prime} \boldsymbol{\Sigma}^{-1} \mathbf{F} \\
\mathbf{F}^{\prime} \boldsymbol{\Sigma}^{-1} \mathbf{D}, & \mathbf{F}^{\prime} \boldsymbol{\Sigma}^{-1} \mathbf{F}
\end{array}\right)^{-1}=\left(\begin{array}{ll}
11, & 12 \\
\hline 21, & 22
\end{array}\right)
$$

It is valid that

$$
\begin{aligned}
21= & -\left[\mathbf{F}^{\prime} \boldsymbol{\Sigma}^{-1} \mathbf{F}-\mathbf{F}^{\prime} \boldsymbol{\Sigma}^{-1} \mathbf{D}\left(\mathbf{W}^{-1}+\mathbf{D}^{\prime} \boldsymbol{\Sigma}^{-1} \mathbf{D}\right)^{-1} \mathbf{D}^{\prime} \boldsymbol{\Sigma}^{-1} \mathbf{F}\right]^{-1} \mathbf{F}^{\prime} \boldsymbol{\Sigma}^{-1} \mathbf{D} \\
& \times\left(\mathbf{W}^{-1}+\mathbf{D}^{\prime} \boldsymbol{\Sigma}^{-1} \mathbf{D}\right)^{-1}, \\
22= & {\left[\mathbf{F}^{\prime} \boldsymbol{\Sigma}^{-1} \mathbf{F}-\mathbf{F}^{\prime} \boldsymbol{\Sigma}^{-1} \mathbf{D}\left(\mathbf{W}^{-1}+\mathbf{D}^{\prime} \boldsymbol{\Sigma}^{-1} \mathbf{D}\right)^{-1} \mathbf{D}^{\prime} \boldsymbol{\Sigma}^{-1} \mathbf{F}\right]^{-1} } \\
= & {\left[\mathbf{F}^{\prime}\left(\boldsymbol{\Sigma}+\mathbf{D} \mathbf{W D}^{\prime}\right)^{-1} \mathbf{F}\right]^{-1} . }
\end{aligned}
$$

Thus

$$
21=-\left[\mathbf{F}^{\prime}\left(\boldsymbol{\Sigma}+\mathbf{D W D}^{\prime}\right)^{-1} \mathbf{F}\right]^{-1} \mathbf{F}^{\prime} \boldsymbol{\Sigma}^{-1} \mathbf{D}\left(\mathbf{W}^{-1}+\mathbf{D}^{\prime} \boldsymbol{\Sigma}^{-1} \mathbf{D}\right)^{-1}
$$
and

$$
\begin{aligned}
\tilde{\mathbf{b}}= & {\left[\mathbf{F}^{\prime}\left(\boldsymbol{\Sigma}+\mathbf{D W} \mathbf{D}^{\prime}\right)^{-1} \mathbf{F}\right]^{-1}\left\{\mathbf{F}^{\prime} \boldsymbol{\Sigma}^{-1} \mathbf{Y}-\mathbf{F}^{\prime} \boldsymbol{\Sigma}^{-1} \mathbf{D}\left(\mathbf{W}^{-1}+\mathbf{D}^{\prime} \boldsymbol{\Sigma}^{-1} \mathbf{D}\right)^{-1}\right.} \\
& \left.\times\left(\mathbf{W}^{-1} \widehat{\boldsymbol{\Theta}}+\mathbf{D}^{\prime} \boldsymbol{\Sigma}^{-1} \mathbf{Y}\right)\right\} \\
= & {\left[\mathbf{F}^{\prime}\left(\boldsymbol{\Sigma}+\mathbf{D W} \mathbf{D}^{\prime}\right)^{-1} \mathbf{F}\right]^{-1}\left\{\mathbf{F}^{\prime} \boldsymbol{\Sigma}^{-1} \mathbf{Y}-\mathbf{F}^{\prime}\left(\boldsymbol{\Sigma}+\mathbf{D W} \mathbf{D}^{\prime}\right)^{-1} \mathbf{D W}\right.} \\
& \left.\times\left(\mathbf{W}^{-1} \widehat{\boldsymbol{\Theta}}+\mathbf{D}^{\prime} \boldsymbol{\Sigma}^{-1} \mathbf{Y}\right)\right\} \\
= & {\left[\mathbf{F}^{\prime}\left(\boldsymbol{\Sigma}+\mathbf{D W} \mathbf{D}^{\prime}\right)^{-1} \mathbf{F}\right]^{-1}\left\{\mathbf{F}^{\prime} \boldsymbol{\Sigma}^{-1} \mathbf{Y}-\mathbf{F}^{\prime}\left(\boldsymbol{\Sigma}+\mathbf{D W} \mathbf{D}^{\prime}\right)^{-1} \mathbf{D} \widehat{\boldsymbol{\Theta}}\right.} \\
& \left.-\mathbf{F}^{\prime}\left(\boldsymbol{\Sigma}+\mathbf{D W} \mathbf{D}^{\prime}\right)^{-1}\left(\boldsymbol{\Sigma}+\mathbf{D W D}^{\prime}-\boldsymbol{\Sigma}\right) \boldsymbol{\Sigma}^{-1} \mathbf{Y}\right\} \\
= & {\left[\mathbf{F}^{\prime}\left(\boldsymbol{\Sigma}+\mathbf{D W} \mathbf{D}^{\prime}\right)^{-1} \mathbf{F}\right]^{-1} \mathbf{F}^{\prime}\left(\boldsymbol{\Sigma}+\mathbf{D W} \mathbf{D}^{\prime}\right)^{-1}(\mathbf{Y}-\mathbf{D} \widehat{\boldsymbol{\Theta}}) . }
\end{aligned}
$$

The expression for $\operatorname{Var}(\tilde{\mathbf{b}})$ is obvious. (For more detail, see [4].) This preliminary estimator $\tilde{\mathbf{b}}$ cannot be considered satisfactory in the state system, because of the structure of its covariance matrix $\left[\mathbf{F}^{\prime}(\boldsymbol{\Sigma}\right.$ $\left.\left.+\mathbf{D W D}^{\prime}\right)^{-1} \mathbf{F}\right]^{-1}$. It produces non-admissibly large dispersions in an estimation of distances among the characteristic points of the new structure. The pro- blem is also how to determine the coordinates of the non-characteristic points of the new structure in the state system. The solution is as follows:

We shall determine the shift and the rotation of the inner coordinate sys- tem in such a way that the new location of the points $\mathrm{P}_{1}, \ldots, \mathrm{P}_{\mathrm{k}}$ approximates the vector $\tilde{\mathbf{b}}$, i.e.,

$$
\widehat{\mathbf{b}}_{i}=\left(\begin{array}{c}
\widehat{t}_{1} \\
\widehat{t_{2}}
\end{array}\right)+\left(\begin{array}{cc}
\widehat{\cos \varphi}, & \widehat{\sin \varphi} \\
-\widehat{\sin \varphi}, & \widehat{\cos \varphi}
\end{array}\right) \mathbf{a}_{i}, i=1, \ldots, k,
$$

whereas the shift $\left(\begin{array}{c}\widehat{t}_{1} \\ \widehat{t}_{2}\end{array}\right)$ and the matrix of the rotation $\left(\begin{array}{cc}\widehat{\cos \varphi}, & \widehat{\sin \varphi} \\ -\widehat{\sin \varphi}, \widehat{\cos \varphi}\end{array}\right)$ will be optimum in the sense of the least-squares theory. This transformation is linear and conforms, and it does not change the distances among the points $P_{i}$ and $P_{j}$.

Let

$$
\begin{aligned}
& \mathbf{T}_{i}=\left(\begin{array}{rrrr}
1, & 0, & a_{i, 1}, & a_{i, 2} \\
0, & 1, & -a_{i, 2}, & a_{i, 1}
\end{array}\right), i=1, \ldots, k, \\
& c=\cos \varphi=c_{0}+\delta c, s=\sin \varphi=s_{0}+\delta s, \\
& t_{1}=t_{1,0}+\delta t_{1}, t_{2}=t_{2,0}+\delta t_{2},
\end{aligned}
$$

where $t_{1,0}, t_{2,0}, c_{0}, s_{0}$ are the approximate values. After the linearization we obtain the following linear model with the type I constraints (for more detail, see [1]).

$$
\begin{aligned}
\boldsymbol{\eta} & =\left(\begin{array}{c}
\tilde{\mathbf{b}}_{1} \\
\vdots \\
\tilde{\mathbf{b}}_{k}
\end{array}\right)-\left(\begin{array}{c}
\mathbf{T}_{1} \\
\vdots \\
\mathbf{T}_{k}
\end{array}\right)\left(\begin{array}{c}
t_{1,0} \\
t_{2,0} \\
c_{0} \\
s_{0}
\end{array}\right) \\
& \sim 2 k\left[\left(\begin{array}{c}
\mathbf{T}_{1} \\
\vdots \\
\mathbf{T}_{k}
\end{array}\right)\left(\begin{array}{c}
\delta t_{1} \\
\delta t_{2} \\
\delta c \\
\delta s
\end{array}\right),\left[\mathbf{F}^{\prime}\left(\boldsymbol{\Sigma}+\mathbf{D W D}^{\prime}\right)^{-1} \mathbf{F}\right]^{-1}\right],
\end{aligned}
$$

with the constraints

$$
c_{0} \delta c+s_{0} \delta s=0
$$

(the term $(\delta c)^{2}+(\delta s)^{2}$ is ignored).

Let

$$
\mathbf{S}=\left[\mathbf{F}^{\prime}\left(\boldsymbol{\Sigma}+\mathbf{D W D}^{\prime}\right)^{-1} \mathbf{F}\right]^{-1}, \quad \mathbf{X}=\left(\begin{array}{c}
\mathbf{T}_{1} \\
\vdots \\
\mathbf{T}_{k}
\end{array}\right)
$$

Lemma 2.2 The BLUEs of the quantities $t_{1}, t_{2}, \cos \varphi, \sin \varphi$ are $\widehat{t}_{1}=$ $t_{1,0}+\widehat{\delta t}_{1}, \widehat{t}_{2}=t_{2,0}+\widehat{\delta t_{2}}, \widehat{\cos \varphi}=c_{0}+\widehat{\delta c}, \widehat{\sin \varphi}=s_{0}+\widehat{\delta s}$, where

$$
\begin{aligned}
& \left(\begin{array}{c}
\widehat{\delta t_{1}} \\
\frac{\delta t_{2}}{\delta c} \\
\widehat{\delta s}
\end{array}\right)=\mathbf{C}^{-1} \mathbf{X}^{\prime} \mathbf{S}^{-1} \boldsymbol{\eta}-\mathbf{C}^{-1} \mathbf{B}^{\prime}\left(\mathbf{B} \mathbf{C}^{-1} \mathbf{B}^{\prime}\right)^{-1} \mathbf{B} \mathbf{C}^{-1} \mathbf{X}^{\prime} \mathbf{S}^{-1} \boldsymbol{\eta}, \\
& \begin{aligned}
& \mathbf{C}=\mathbf{X}^{\prime} \mathbf{S}^{-1} \mathbf{X}, \quad \mathbf{B}=\left(0,0, c_{0}, s_{0}\right), \\
& \operatorname{Var}\left(\begin{array}{c}
\widehat{\delta t_{1}} \\
\frac{\widehat{\delta t_{2}}}{\widehat{\delta c}} \\
\widehat{\delta s}
\end{array}\right)=\mathbf{C}^{-1}-\mathbf{C}^{-1} \mathbf{B}^{\prime}\left(\mathbf{B C}^{-1} \mathbf{B}^{\prime}\right)^{-1} \mathbf{B} \mathbf{C}^{-1}=\mathbf{V} .
\end{aligned}
\end{aligned}
$$

For the proof, see [4].

QED

The resulting location of the point $\mathrm{Pi}$ in the state system is given by the vector

$$
\widehat{\mathbf{b}}_{i}=\left(\begin{array}{ccc}
\widehat{t}_{1}, & 0, \widehat{\cos \varphi}, \widehat{\sin \varphi} \\
0, \widehat{t_{2}} & -\widehat{\sin \varphi}, \widehat{\cos \varphi}
\end{array}\right) \mathbf{a}_{i}=\mathbf{T}_{i}\left(\begin{array}{c}
\widehat{t}_{1} \\
\widehat{t_{2}} \\
\widehat{\cos \varphi} \\
\sin \varphi
\end{array}\right), i=1, \ldots, k .
$$

The expression for $\widehat{\mathbf{b}}_{i}$ can be rewritten as follows

$$
\begin{gathered}
\widehat{\mathbf{b}}_{i}=\mathbf{T}_{i}\left\{\mathbf{I}-\mathbf{C}^{-1} \mathbf{B}^{\prime}\left[\mathbf{B} \mathbf{C}^{-1} \mathbf{B}^{\prime}\right]^{-1} \mathbf{B}\right\} \mathbf{C}^{-1} \mathbf{X}^{\prime} \mathbf{S}^{-1}\left(\tilde{\mathbf{b}}-\mathbf{b}_{0}\right) \\
=\mathbf{T}_{i}\left\{\mathbf{I}-\mathbf{C}^{-1} \mathbf{B}^{\prime}\left[\mathbf{B} \mathbf{C}^{-1} \mathbf{B}^{\prime}\right]^{-1} \mathbf{B}\right\} \mathbf{C}^{-1} \mathbf{X}^{\prime} \mathbf{F}^{\prime}\left(\boldsymbol{\Sigma}+\mathbf{D W D}^{\prime}\right)^{-1}(\mathbf{Y}-\mathbf{D} \widehat{\Theta}),
\end{gathered}
$$

where

$$
\mathbf{b}_{0}=\left(\begin{array}{c}
\mathbf{T}_{1} \\
\vdots \\
\mathbf{T}_{k}
\end{array}\right)\left(\begin{array}{c}
t_{1,0} \\
t_{2,0} \\
c_{0} \\
s_{0}
\end{array}\right)
$$

The estimator $\widehat{\mathbf{b}}$ is the final estimator of the coordinates of the characteristic points of the steructure in the state coordinate system. The transformation can be used for the non-characteristic points of the structure as well.

Let

$$
\mathbf{Z}_{i}=\mathbf{T}_{i}\left\{\mathbf{I}-\mathbf{C}^{-1} \mathbf{B}^{\prime}\left[\mathbf{B} \mathbf{C}^{-1} \mathbf{B}^{\prime}\right]^{-1} \mathbf{B}\right\} \mathbf{C}^{-1} \mathbf{X}^{\prime} \mathbf{F}^{\prime}\left(\boldsymbol{\Sigma}+\mathbf{D W D}^{\prime}\right)^{-1} .
$$


Lemma 2.3 The covariance matrix of the vector $\left(\widehat{\boldsymbol{\Theta}}^{\prime}, \widehat{\mathbf{b}}_{i}^{\prime}\right)^{\prime}$ is

$$
\operatorname{cov}\left(\widehat{\boldsymbol{\Theta}}, \widehat{\mathbf{b}}_{i}\right)=-\mathbf{W} \mathbf{D}^{\prime} \mathbf{Z}_{i}^{\prime}
$$

and the covariance matrix of the vector $\left(\widehat{\mathbf{b}}_{i}^{\prime}, \widehat{\mathbf{b}}_{j}^{\prime}\right)^{\prime}$

$$
\operatorname{cov}\left(\widehat{\mathbf{b}}_{i}, \widehat{\mathbf{b}}_{j}\right)=\mathbf{T}_{i} \mathbf{V} \mathbf{T}_{j}^{\prime}
$$

Thus

$$
\operatorname{Var}\left(\begin{array}{c}
\widehat{\boldsymbol{\Theta}} \\
\widehat{\mathbf{b}}_{1} \\
\vdots \\
\widehat{\mathbf{b}}_{k}
\end{array}\right)=\left(\begin{array}{cccc}
\mathbf{W}, & -\mathbf{W D}^{\prime} \mathbf{Z}_{1}^{\prime}, & \ldots, & -\mathbf{W D}^{\prime} \mathbf{Z}_{k}^{\prime} \\
-\mathbf{Z}_{1} \mathbf{D W}, & \mathbf{T}_{1} \mathbf{V} \mathbf{T}_{1}^{\prime}, & \ldots, & \mathbf{T}_{1} \mathbf{V} \mathbf{T}_{k}^{\prime} \\
\ldots \ldots \ldots \ldots \ldots \ldots \ldots \ldots \ldots \ldots \ldots \ldots \ldots . \\
-\mathbf{Z}_{k} \mathbf{D W}, & \mathbf{T}_{k} \mathbf{V} \mathbf{T}_{1}^{\prime}, & \ldots, & \mathbf{T}_{k} \mathbf{V} \mathbf{T}_{k}^{\prime}
\end{array}\right)
$$

Proof. Since $\widehat{\mathbf{b}}_{i}=\mathbf{Z}_{i}(\mathbf{Y}-\mathbf{D} \widehat{\boldsymbol{\Theta}})$, it is valid that

$$
\operatorname{cov}\left(\widehat{\Theta}, \widehat{\mathbf{b}}_{i}\right)=-\mathbf{W D}^{\prime} \mathbf{Z}_{i}^{\prime}, i=1, \ldots, k,
$$

and

$$
\operatorname{cov}\left(\widehat{\mathbf{b}}_{i}, \widehat{\mathbf{b}}_{j}\right)=\mathbf{Z}_{i} \operatorname{Var}(\mathbf{Y}-\mathbf{D} \widehat{\boldsymbol{\Theta}}) \mathbf{Z}_{j}^{\prime}
$$

$=\mathbf{T}_{i} \mathbf{V X}^{\prime} \mathbf{F}^{\prime}\left(\boldsymbol{\Sigma}+\mathbf{D W D}^{\prime}\right)^{-1}\left(\boldsymbol{\Sigma}+\mathbf{D W D}^{\prime}\right) \times\left(\boldsymbol{\Sigma}+\mathbf{D W D}^{\prime}\right)^{-1} \mathbf{F X V T}_{j}^{\prime}$

$$
=\mathrm{TVX}^{\prime} \mathbf{S}^{-1} \mathbf{X V T}_{j}^{\prime}=\mathbf{T}_{i} \mathbf{V C V T}_{j}^{\prime}=\mathbf{T}_{i} \mathbf{V T}_{j}^{\prime},
$$

since

$$
\begin{aligned}
\mathbf{V C V}= & \left\{\mathbf{C}^{-1}-\mathbf{C}^{-1} \mathbf{B}^{\prime}\left[\mathbf{B C}^{-1} \mathbf{B}^{\prime}\right]^{-1} \mathbf{B}^{\prime} \mathbf{C}^{-1}\right\} \mathbf{C} \\
& \times\left\{\mathbf{C}^{-1}-\mathbf{C}^{-1} \mathbf{B}^{\prime}\left[\mathbf{B C}^{-1} \mathbf{B}^{\prime}\right]^{-1} \mathbf{B}^{\prime} \mathbf{C}^{-1}\right\}=V .
\end{aligned}
$$

Theorem 2.4 The distances among the characteristic points $P_{1}, \ldots$, $P_{k}$ with the coordinate vectors $\mathbf{a}_{1}, \ldots, \mathbf{a}_{\mathrm{k}}$ and the points $C_{1}, \ldots, \mathrm{C}_{1}$, with the internal coordinate vectors $c_{1}, \ldots, c_{1}$ are not changed by the transformation with the parameters

i.e.,

$$
\widehat{t_{1}}, \widehat{t_{2}}, \widehat{\cos \varphi}, \widehat{\sin \varphi},
$$

$$
\operatorname{Var}\left(\left\|\widehat{\mathbf{b}}_{i}-\widehat{\mathbf{d}}_{j}\right\|^{2}\right)=0
$$

where $\widehat{\mathbf{d}}_{j}$ is the transformed location of point $C_{j}$.

Proof. The location of point $P_{i}$ with the coordinate vector $\mathbf{a}_{i}$ and point $C_{j}$ with coordinate vector $\mathbf{c}_{i}$ after transformation is given by the vectors

$$
\widehat{\mathbf{b}}_{i}=\left(\mathbf{I}, \mathbf{A}_{i}\right)\left(\begin{array}{c}
\widehat{t}_{1} \\
\widehat{t_{2}} \\
\widehat{\cos \varphi} \\
\sin \varphi
\end{array}\right), \quad \widehat{\mathbf{d}}_{i}=\left(\mathbf{I}, \mathbf{C}_{j}\right)\left(\begin{array}{c}
\widehat{t}_{1} \\
\widehat{t_{2}} \\
\widehat{\cos \varphi} \\
\sin \varphi
\end{array}\right),
$$

where

$$
\mathbf{A}_{i}=\left(\begin{array}{rr}
a_{i, 1}, & a_{i, 2} \\
-a_{i, 2}, & a_{i, 1}
\end{array}\right), \quad \mathbf{C}_{j}=\left(\begin{array}{rr}
c_{j, 1}, & c_{j, 2} \\
-c_{j, 2}, & c_{j, 1}
\end{array}\right)
$$

It means that

$$
\begin{aligned}
& \left\|\widehat{\mathbf{b}}_{i}-\widehat{\mathbf{d}}_{j}\right\|^{2}=\left\|\left[\left(\mathbf{I}, \mathbf{A}_{i}\right)-\left(\mathbf{I}, \mathbf{C}_{j}\right)\right]\left(\begin{array}{c}
\widehat{t_{1}} \\
\widehat{\widehat{t}_{2}} \\
\widehat{\cos \phi} \\
\widehat{\sin \phi}
\end{array}\right)\right\|^{2} \\
& =(\widehat{\cos \phi}, \widehat{\sin \phi})\left(\mathbf{A}_{i}-\mathbf{C}_{j}\right)^{\prime}\left(\mathbf{A}_{i}-\mathbf{C}_{j}\right)\left(\begin{array}{c}
\widehat{\cos \phi} \\
\widehat{\sin \phi}
\end{array}\right) \\
& =\left(a_{i, 1}-c_{j, 1}\right)^{2}+\left(a_{i, 2}-c_{j, 2}\right)^{2}=\left\|\mathbf{a}_{i}-\mathbf{c}_{j}\right\|^{2} .
\end{aligned}
$$

Remark 2.5 Since the distances among the points $P_{1}, \ldots, P_{k}$, $C_{1}, \ldots, C_{l}$ are not changed after the transformation, the angles do not change as well, i.e., they are error-free. However, it is necessary to remark that the covariance matrix of the estimators of the coordinates of the characteristic and non-characteristic points of the new structure is not a zero matrix, i.e., the dispersions of the coordinate estimators are not zero as well. The dispersions are of the same size as the dispersions of the coordinates of points $B_{1}, \ldots, B_{N}$.

Remark 2.6 There is also another approach to the determination of the state coordinates of the characteristic points of the structure. However, it is optimum in another sense. The question is about the $H$ - optimum estimator (for more detail, see [6]).

\section{EXAMPLE}

The following example shows that the idea given in the preceding sections can be used in the case of levelling networks as well.

Let a levelling network consist of the points $B_{1}, B_{2}$ with estimated heights equal to $\widehat{\Theta}_{1}$ and $\widehat{\Theta}_{2}$, respectively,

$$
\left(\begin{array}{c}
\widehat{\Theta}_{1} \\
\widehat{\Theta}_{2}
\end{array}\right) \sim_{2}\left[\left(\begin{array}{c}
\Theta_{1} \\
\Theta_{2}
\end{array}\right),\left(\begin{array}{ll}
w_{1,1}, & w_{1,2} \\
w_{2,1}, & w_{2,2}
\end{array}\right)\right] .
$$

The points $P_{1}, P_{2}$ are of unknown heights $b_{1}$ and $b_{2}$, respectively. Let a measurement of the unknown heights be given by the following model:

$$
\left(\begin{array}{l}
Y_{1} \\
Y_{2}
\end{array}\right) \sim_{2}\left[\left(\begin{array}{rrrr}
-1, & 0, & 1, & 0 \\
0, & 1, & 0, & -1
\end{array}\right)\left(\begin{array}{c}
\Theta_{1} \\
\Theta_{2} \\
b_{1} \\
b_{2}
\end{array}\right),\left(\begin{array}{cc}
\tau_{1}, & 0 \\
0, & \tau_{2}
\end{array}\right)\right],
$$

i.e.

$$
\left(\begin{array}{c}
\widehat{\boldsymbol{\Theta}} \\
\mathbf{Y}
\end{array}\right) \sim_{4}\left[\left(\begin{array}{rrrr}
1, & 0, & 0, & 0 \\
0, & 1, & 0, & 0 \\
-1, & 0, & 1, & 0 \\
0, & 1, & 0, & -1
\end{array}\right),\left(\begin{array}{cccc}
w_{1,1}, & w_{1,2}, & 0, & 0 \\
w_{2,1}, & w_{2,2}, & 0, & 0 \\
0, & 0, & \tau_{1}, & 0 \\
0, & 0, & 0, & \tau_{2}
\end{array}\right)\right] .
$$

Thus (Lemma 2.1)

$$
\begin{aligned}
&\left(\begin{array}{l}
\tilde{b}_{1} \\
\tilde{b}_{2}
\end{array}\right)=\left\{( \begin{array} { r r } 
{ 1 , } & { 0 } \\
{ 0 , } & { - 1 }
\end{array} ) \left[\left(\begin{array}{rr}
\tau_{1}, & 0 \\
0, & \tau_{2}
\end{array}\right)+\left(\begin{array}{rr}
-1, & 0 \\
0, & 1
\end{array}\right)\left(\begin{array}{ll}
w_{1,1}, & w_{1,2} \\
w_{2,1}, & w_{2,2}
\end{array}\right)\right.\right. \\
&\left.\left.\times\left(\begin{array}{rr}
-1, & 0 \\
0, & 1
\end{array}\right)\right]^{-1}\left(\begin{array}{rr}
1, & 0 \\
0, & -1
\end{array}\right)\right\}^{-1} \\
& \times\left(\begin{array}{rr}
1, & 0 \\
0, & -1
\end{array}\right)\left[\left(\begin{array}{rr}
\tau_{1}, & 0 \\
0, & \tau_{2}
\end{array}\right)+\left(\begin{array}{rr}
-1, & 0 \\
0, & 1
\end{array}\right)\left(\begin{array}{rr}
w_{1,1}, & w_{1,2} \\
w_{2,1}, & w_{2,2}
\end{array}\right)\left(\begin{array}{rr}
-1, & 0 \\
0, & 1
\end{array}\right)\right]^{-1} \\
& \times\left[\left(\begin{array}{c}
Y_{1} \\
Y_{2}
\end{array}\right)-\left(\begin{array}{rr}
-1, & 0 \\
0, & 1
\end{array}\right)\left(\begin{array}{c}
\widehat{\Theta}_{1} \\
\widehat{\Theta}_{2}
\end{array}\right)\right]=\left(\begin{array}{c}
\widehat{\Theta}_{1}+Y_{1} \\
\widehat{\Theta}_{2}-Y_{2}
\end{array}\right)
\end{aligned}
$$

and

$\operatorname{Var}\left(\begin{array}{l}\tilde{b}_{1} \\ \tilde{b}_{2}\end{array}\right)=\left(\begin{array}{ll}\sigma_{1,1}, & \sigma_{1,2} \\ \sigma_{2,1}, & \sigma_{2,2}\end{array}\right)=\left(\begin{array}{cc}w_{1,1}+\tau_{1}, & w_{1,2} \\ w_{2,1}, & w_{2,2}+\tau_{2}\end{array}\right)=\mathbf{\Sigma}$

If $b_{2}-b_{1}=a$ is known, then an analogy of the Helmert transformation in $1 \mathrm{D}$ is

$$
\left(\begin{array}{l}
\tilde{b}_{1} \\
\tilde{b}_{2}
\end{array}\right) \sim_{2}\left[\left(\begin{array}{l}
0 \\
a
\end{array}\right)+\left(\begin{array}{l}
1 \\
1
\end{array}\right) \beta, \Sigma\right],
$$

i.e. $b_{1}=\beta$ and $b_{2}=\beta+a$.

The BLUE of the transformation parameter $\beta$ in this model is

$$
\begin{gathered}
\widehat{\beta}=\left[(1,1) \boldsymbol{\Sigma}^{-1}\left(\begin{array}{c}
1 \\
1
\end{array}\right)\right]^{-1}(1,1) \boldsymbol{\Sigma}^{-1}\left(\begin{array}{c}
\tilde{b}_{1} \\
\tilde{b}_{2}-a
\end{array}\right) \\
=\frac{1}{\sigma_{1,1}+\sigma_{2,2}-2 \sigma_{1,2}}\left[\left(\sigma_{2,2}-\sigma_{1,2}\right) \tilde{b}_{1}+\left(\sigma_{1,1}-\sigma_{1,2}\right)\left(\tilde{b}_{2}-a\right)\right] .
\end{gathered}
$$



Thus
\[ \begin{array}{l}\widehat{b}_{1}=\widehat{\beta}, \\ \widehat{b}_{2}=\widehat{\beta}+a=\frac{1}{\sigma_{1,1}+\sigma_{2,2}-2 \sigma_{1,2}}\left[\left(\tilde{b}_{1}+a\right)\left(\sigma_{2,2}-\sigma_{1,2}\right)+\tilde{b}_{2}\left(\sigma_{1,1}-\sigma_{1,2}\right)\right] . \quad \text { the covariance matrix Var }\left(\begin{array}{l}\widehat{\Theta}_{1} \\ \widehat{\Theta}_{2} \\ \widehat{b}_{1} \\ \widehat{b}_{2}\end{array}\right) \text { can be written in the form }\end{array} \]

Since

$$
\begin{aligned}
\operatorname{cov}\left(\widehat{\Theta}_{1}, \widehat{b}_{1}\right)= & \frac{1}{w_{1,1}+w_{2,2}+\tau_{1}+\tau_{2}-2 w_{1,2}}\left[\left(w_{2,2}+\tau_{2}-w_{1,2}\right) w_{1,1}\right. \\
& \left.+\left(w_{1,1}+\tau_{1}-w_{1,2}\right) w_{1,2}\right]=\operatorname{cov}\left(\widehat{\Theta}_{1}, \widehat{b}_{2}\right)=c_{1,2} \\
\operatorname{cov}\left(\widehat{\Theta}_{2}, \widehat{b}_{1}\right)= & \frac{1}{w_{1,1}+w_{2,2}+\tau_{1}+\tau_{2}-2 w_{1,2}}\left[\left(w_{2,2}+\tau_{2}-w_{1,2}\right) w_{1,2}\right. \\
& \left.+\left(w_{1,1}+\tau_{1}-w_{1,2}\right) w_{2,2}\right]=\operatorname{cov}\left(\widehat{\Theta}_{2}, \widehat{b}_{2}\right)=c_{2,2},
\end{aligned}
$$$$
\operatorname{Var}\left(\begin{array}{c}
\widehat{\Theta}_{1} \\
\widehat{\Theta}_{2} \\
\widehat{b}_{1} \\
\widehat{b}_{2}
\end{array}\right)=\left(\begin{array}{llll}
w_{1,1}, & w_{1,2}, & c_{1,1}, & c_{1,1} \\
w_{2,1}, & w_{2,2}, & c_{2,1}, & c_{2,1} \\
c_{1,1}, & c_{2,1}, & 1, & 1 \\
c_{1,1}, & c_{2,1}, & 1, & 1
\end{array}\right)
$$

where

$$
1=\frac{\left(w_{1,1}+\tau_{1}\right)\left(w_{2,2}+\tau_{2}\right)-w_{1,2}^{2}}{w_{1,1}+w_{2,2}+\tau_{1}+\tau_{2}-2 w_{1,2}} .
$$

\section{REFERENCES}

[1] Fišerová, E. - Kubáček, L. - Kunderová, P. (2007) Linear Statistical Models - Regularity and Singularities, Academia, Prague 2007.

[2] Kubáček, L. (1993) Two stage linear models with constraints, Math. Slovaca 43, pp. 643-658.

[3] Kubáček, L. (1994) Štatistické modely pripojovacích meraní (Statistical models of connecting measurements), In: Petrovič, J. (ed.) Modernizácia geodetických základov Slovenska, VÚGK Bratislava, pp. 28-40.

[4] Kubáček, L. - Kubáčková, L. - Volaufová, J. (1995) Statistical Models with Linear Structures, Veda, Bratislava.
[5] Kubáček, L. - Kubáčková, L. - Ševčík, J. (2002) Linear conform transformation: errors in both coordinate systems, Math. Slovaca 47 , pp. $361-380$.

[6] Kubáček, L. - Marek, J. (2005) Partial optimum estimator in two stage regression model with constraints and a problem of equivalence, Math. Slovaca 55, pp. 477-494.

[7] Kubáček, L. (2009) Statistics and 3D transformation, In: Holota, P. (ed.) Mission and Passion Science. Volum dedicated to Milan Burša on the occasion of his 80 birth, Czech National Committee of Geodesy and Geophysics. Prague, pp. 159-167. 